sätzlich erhöhten Chemotherapiesensitivität im Falle von TIL.

Vor diesem Hintergrund sind die Daten von Dieci und Kollegen besonders interessant, da hier kein Zusammenhang mit einer erhöhten Wirksamkeit für die anthrazyklinhaltige Chemotherapie gesehen wurde. Die Forscher selbst begründen diese Ergebnisse jedoch korrekterweise eher methodisch denn biologisch. Sie verweisen darauf, dass a) die verwendeten Anthrazyklin-Dosen nicht dem aktuellen Standard entsprechen und $b$ ) in dieser Studie weder Taxane noch Platinsalze verwendet wurden. Höhere Anthrazyklin-Dosen könnten erforderlich sein, um eine entsprechende Tumor-Immunsystem-Wirkung auszulösen, die sich in einem prädiktiven Unterschied widerspiegeln könnte. Vor diesem Hintergrund lässt sich demzufolge keine substanz-spezifische prädiktive Aussagekraft von TIL annehmen.

Grundsätzlich ist darauf hinzuweisen, dass die Bestimmung von TIL noch nicht ausrei- chend standardisiert ist [Salgado $\mathrm{R}$ et al. Ann Oncol. 2015;26(2):259-71]. Auch sind bis dato keine wissenschaftlichen Ergebnisse verfügbar, auf deren Basis sich eine klinische Konsequenz aus erhöhten oder verminderten TIL ableiten ließe.

Neben der alleinigen prognostischen Bedeutung sind TIL als Ansatz für neue immunmodulatorische Substanzen interessant und stellen daher einen wichtigen Bestandteil der aktuellen Forschung dar. Zielgerichtete Substanzen, z.B. Inhibitoren des Checkpointmoleküls PD-1 („programmed cell death 1“) und seines Liganden 1 (PD-L1), haben beim TNBC beachtliche Wirksamkeit zeigen können [Nanda R et al. SABCS. 2014; Abstr S1-09] und werden derzeit in verschiedenen klinischen Studien systematisch auf ihre Wirksamkeit hin untersucht.

Zusammenfassend ist festzustellen, dass die vorliegende Studie die starke prognostische Rolle der TIL beim TNBC und HER2positiven Mammakarzinom unterstreicht. Eine signifikante Interaktion von TIL und Antrazyklinen war nicht nachweisbar. Um die genauen immunologischen Mechanismen bei den verschiedenen Mammakarzinom-Subgruppen besser zu verstehen, sind weitere Studien und Untersuchungen notwendig. Ziel dieser Studien sollte es sein, neue Substanzen zu entwickeln, die eine effiziente antitumorale Immunantwort stimulieren können.

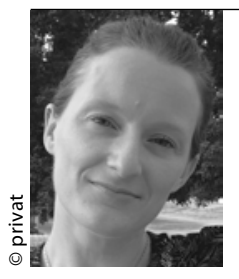

PD Dr. med.

Cornelia Liedtke Klinik für Frauenheilkunde und Geburtshilfe Universitätsklinikum Schleswig-Holstein Campus Lübeck Cornelia.Liedtke@uksh.de

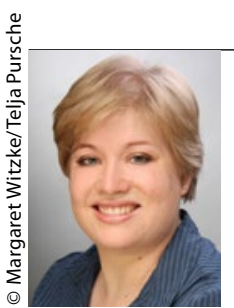

Dr. med. Telja Pursche Klinik für Frauenheilkunde und Geburtshilfe Universitätsklinikum Schleswig-Holstein Campus Lübeck telja.pursche@uksh.de

\section{PET/CT-Risikostratifizierung bei fortgeschrittenem Brustkrebs scheint möglich}

\section{Die Prognoseabschätzung mittels [ $\left.{ }^{18} \mathrm{~F}\right]$ Fluorodeoxyglukose-Positronenemis- sionstomografie (FDG-PET/CT) hat bei Brustkrebs in bisherigen Studien nicht überzeugt. Jetzt wurde bei fortgeschrittener Erkrankung ein neuer Messwert mit einem Schwellenwert etabliert, der prognostisch relevant ist.}

$B$ asis hierfür war die retrospektive Analyse der PET/CT-Scans von 240 Patientinnen mit Brustkrebs im Stadium III oder IV nach den Kriterien des American Joint Committee on Cancer (AJCC). Ausgewertet wurden klinisch-pathologische Faktoren und metabolische Parameter des Primärtumors: der maximale standardisierte Aufnahmewert $\left(\mathrm{SUV}_{\text {max }}\right)$, das metabolische Tumorvolumen (MTV) und die Gesamtglykolyse des Tumors (TLG). Der SUV $\mathrm{V}_{\max }$ repräsentiert die intensivste FDG-Aufnahme im Tumor. Die TLG wird durch Multiplikation der MTV mit dem mittleren SUV kalkuliert und integriert metabolische Aktivität und Tumorvolumen. Deshalb könnte sie eine präzisere Information hinsichtlich der Tumorlast bieten.
Einige Schwellenwerte wurden zur Vorhersage des progressionsfreien Überlebens (PFS) und des Gesamtüberlebens (OS) mithilfe einer zeitabhängigen Grenzwert-Optimierungskurve und einer Cox-Regressionsanalyse verglichen. Multivariat wurden als Schwellenwerte für $S_{U V} V_{\max } 6,0$ und für ein 30\%iges TLG $\left(\mathrm{TLG}_{30 \%}\right) 158 \mathrm{~g}$ festgelegt.

Der mittlere Wert für die $\mathrm{TLG}_{30 \%}$ lag bei Patientinnen mit Brustkrebs im Stadium III bei $405 \mathrm{~g}$, bei einem StadiumIV-Karzinom bei $750 \mathrm{~g}(\mathrm{p}=0,010)$. Als Hochrisikoerkrankungen wurden triple-negative Tumoren und solche mit einem TLG $_{30 \%}$ über $158 \mathrm{~g}$ oder mit beiden Charakteristika definiert. Nicht triplenegative Tumoren und solche mit einem $\mathrm{TLG}_{30 \%}$ des Primärtumors von maximal
158 g waren demnach NiedrigrisikoKarzinome.

Die 5-Jahres-PFS-Rate bei einem Mammakarzinom des AJCC-Stadiums III betrug bei niedrigem Risiko $85 \%$ und bei hohem Risiko $67,5 \%$, bei einem Stadium IV und niedrigem Risiko $45 \%$ und bei hohem Risiko 9\%. Die Rate für das 5-Jahres-OS lag bei Stadium III und niedrigem Risiko bei $91 \%$, bei hohem Risiko bei $69 \%$. Im Stadium IV betrug die 5-Jahres-OS-Rate bei niedrigem Risiko $63 \%$ und bei hohem Risiko $14 \%$. Die Überlebensprognose von Patientinnen mit Stadium III und hohem Risiko war also vergleichbar mit dem im Stadium IV mit niedrigem Risiko $(\mathrm{p}=0,552)$.

Fazit: Die $\mathrm{TLG}_{30 \%}$ in der PET/CT vor Behandlung korrelierte in dieser retrospektiven Auswertung unabhängig von anderen Parametern mit dem PFS und dem OS. Sie könnte helfen, Patientinnen mit fortgeschrittenem Mammakarzinom noch besser hinsichtlich ihrer Prognose zu stratifizieren. Friederike Klein

Chen S et al. Risk stratification in patients with advanced-stage breast cancer by pretreatment [(18) F]FDG PET/CT. Cancer. 2015;121(22):3965-74. 